\title{
USER PROFILE MIGRATING APPLICATION FOR WINDOWS SMALL OFFICE AND HOME USERS
}

\author{
Veronika Szucs $^{1}$ and Martin Erdely ${ }^{2}$ \\ University of Pannonia, 10 Egyetem str. veszprém, Hungary
}

\begin{abstract}
The purpose of the manuscript is to present an application that is designed to ensure that user accounts are moved in the context of computer replacement or reinstallation in both home and enterprise environments. In a small office or home office environment it is important to be able to move user account's data between different computers or save and restore all the important data, document after system reinstallation. It is a natural demand that users would like to select the desired user accounts that want to save or restore, and the non-system administrator qualified users usually need a graphical user interface which displays important information for selected accounts and transactions.

When saving user profiles, consideration is given to selecting a secure storage location, saving to external storage or network location. The process also involves compressing and encrypting data to ensure that private and confidential data is handled in accordance with the new GDPR rules (EU 2018).
\end{abstract}

\section{KEYWORDS}

Profile, Backup, Recover, Migration, User, Security

\section{INTRODUCTION}

The program what will be introduced in this paper was designed to give an opportunity to the general computer users to easily backup and restore their own user profiles, while keeping the underlying processes secure and highly customizable. The identified problem is that general users are unsatisfied with their system's health, however they do not actively try to solve the issue due to the time-consuming process of manually saving and restoring user data. The purpose of authors' application is to automate the profile migration, making it possible to finish a complete reinstall or a computer replacement under an hour or two, while keeping all the user settings and personal files.

The following sections will further unfold the planning, the implementation, the difficulties and the thought process behind creating a tool, capable of migrating computer user profiles. Even though such tools are already available at the market, they tend to target only companies and lack any customization. The aim was to create a program that helps managing the general users' profiles, while giving them significant control over the backup and recovering operation.

\section{METHODOLOGY}

\subsection{Defining User Needs (Requirement Specification)}

The main goal was to serve the users' needs. In most user migrating application the users cannot find a wide range of backup options, and significant number of them is designed for professional use only, not for the general users but especially for system administrators (Bila 2012, Kupczyket 2005).

\footnotetext{
${ }^{1}$ szucs@ virt.uni-pannon.hu

2 marty.sniper@gmail.com
} 


\subsection{Available Applications}

Before the planning the authors were performed a small research over the available commercial and free user account migrating applications. The widest range of system administrators uses User State Migration Tool (USMT - Microsoft product) (Jayathilake 2015), PCmover Express from the Laplink (PCmover 2018) software company or AOMEI Backupper (Aomei 2018). The USMT'main disadvantage is that the software's target group is only from the big business companies and does not contain any personal option for the backup and restore process. The PCmover Express has a few settings possibilities, but this can be reached only in commercial version. The AOMEI Bacupper is not to backup user accounts but backup general data from the PC. None of the abovementioned programs provide general information about user profiles such as last login or all user data. Unfortunately, the user cannot save only the selected user profiles with these applications.

The main reason for the new development was to provide the missing features.

\subsection{Software Development Method}

There are many design methods in the world of software development, with which they can design programs more easily and with fewer errors, make it easier to access the functions set out in the specification and provide guidance for integration and testing (Zima 2015).

From the available software development methodologies, the chosen one for the actual development was the V-model based development because by adapting to the user needs, continuous development ensures that the functional requirements and implemented functionalities are consistent. The advantage of the model is that it breaks down the entire design into smaller parts and each section has its own counterpart between the testing stages, so it can easily highlight any errors (V-model 2016, Young 2013).

\section{FUNCTIONAL PLAN}

\subsection{Target Operating System}

The program was planned to run on Windows operating systems, simply because those are the most common systems of all, with the greatest market share (AppiXoft User Profile Management (AppiXoft 2018), Virtualizing Windows10 User Profiles (User Profile 2018, Rankin 2016)). Even with Windows there are significant differences between iterations, so this work is mainly focused on Windows 10, however resolving errors caused by Windows differences should not be a problem. The real challenge would be to make the application platform independent, which would further require tremendous planning, developing and testing, but the number of users with this request are slim. One thing that is worth mentioning though is even if the demand for cross-platform migration at a home environment is not that significant, there is no application currently published at the market, which can achieve this.

\subsection{Functionalities}

Based on the user requirements there are five recognized main part in the application:

1. Assess the state of the system

2. Collecting data from user accounts

3. Backup, restore

3.1 Compression, unpacking

3.1. Encryption, decoding

Functionality-wise the application will ask the users to select whichever profile there is on their pc to backup, the same way it gives them the opportunity to select any user profiles saved to a compressed file by the program to restore. Before backup, the program should offer the option to save the profiles to an external storage device or a network storage, however the main goal is a cloud service, which would simplify the usage for the user, but requires more resources and infrastructure. 


\section{IMPLEMENTATION}

The following section contains a brief description about the implemented functionalities in the application.

\subsection{Asses the State of the System}

The program performs extensive diagnostics. Evaluates the data of all users who have ever logged in, such as: name, username, size of all data stored in user's folders, last login date, account type (guest, user, administrator). After the survey, the program displays the number of users found and the total area they have allocated on a graphical interface, and lists the data in user backups under the data backup tab. These data provide useful information to the person who is about to make the backup about the relevance of the account, the expected time of the backup and the required storage space.

\subsection{Collecting Data from User Accounts}

After the program has been started, it scans the hard disk the system is located on and the registry entries. When the scan has finished, it shows basic information about the number of users found on the computer, the size of each user's personal files and settings, the user's last logon date and overall how much space there is left on the system's drive. This information helps the user estimate the time it takes to complete a backup or a restore operation, furthermore it indicates the storage needs for the data saving process.

\subsection{Backup and Restore}

Backup and restore are the core operations done by the application. At backup after the user selection has been made, the program starts to collect the personal data and settings, ignore all the temporary or unnecessary files and installed programs. During the backup the program saves the data to one compressed file with a unique format and when it is completed some other data gets written into the compressed file for handling and authenticating reasons. Same thing happens at restoring just in a reverse order. The program reads the compressed file and creates the selected users locally. After that it copies the user's personal files and registry entries to the system's drive. When everything is done it should work just like before the backup.

For the application to work securely and flawlessly it is important to use an encryption, otherwise users without permission could steal information about other user's saved profile. Besides the encryption it is optimal to use a compression algorithm too, for the sake of space saving. To build in a well-working encryption is a basic requirement more so if the application will be used in an enterprise environment, and the EU GDPR rule takes it as an obligatory rule in this question.

\subsubsection{Compression, Unpacking}

During the data collection, the files are not saved "raw" by the application but added to a compressed file created on the storage device. This operation has several advantages, such as space saving, systematization and file insertion. The program will use a unique file format and after the compression. In addition, compression requires the use of a so-called lossless compression, an algorithm that can later be converted from the file received to 100 percent of the original files without loss.

\subsubsection{Compression, Unpacking User Data}

Encryption is a must have in today's informational society when handling user information. The software must build trust towards potential customers and must ensure them the safety of their personal belongings. There are plenty encrypting algorithms out there and we chose RSA [ccc], mainly because of the advantages and freedom the public-key encryption gives us.

Compression is not obligatory, but well recommended. It's important to use a so called "lossless" compression algorithm, because we must get the user data back byte by byte and it must match perfectly with the original data. 


\subsubsection{Restoring User Accounts}

The result of the process is a compressed and encrypted file that contains all user data, settings, and essentially every selected account. The users' files can be restored to another computer on request or can be restored to the current computer after reinstallation. The user will be able to select which accounts want to move, and then perform the necessary actions. After the restore, the accounts will look like before the data backup, but they will take up less space because the application does not save any temporary files.

\section{INITIAL TESTING}

Testing was an important part of the development, and even in large programs, many errors can remain hidden for years, until a special event replaces it.

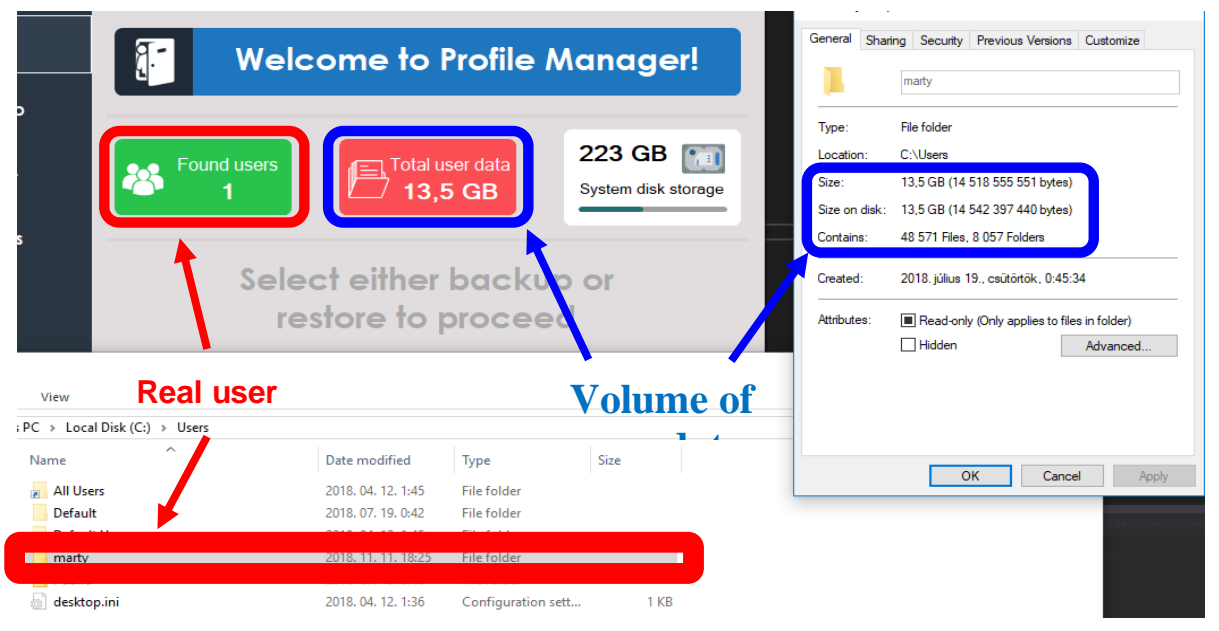

Figure 1. Figure Initial system test - accessing user data

User account management program is not easy to test. There are a lot of data moving, home users usually have only a few users, no great samples, no small or specific errors appear. Fortunately, with the support of Arconic-Köfém Ltd. in Hungary, the application was tested on their business environment. The tests carried out in this way are effective because many user accounts are present on each machine and have been tested on inhomogeneous hardware files. One of the initial tests can be seen in Figure 1, and it presents, that the recognized user accounts in the application are equal to the host operating system presented information. The next interesting question was the amount of user data, and as the Figure 1. shows, the volume of reached data is the same compared to the system presented volume of the user profile's volume.

\section{DISCUSSION}

The application has several enhancements that can be deployed later to be able to perform a wider range of tasks.

Migrating a user on one operating system to another operating system, with all its settings, is not a trivial task and would still be a great demand on the market for an application that can fulfill this role.

The next option would be to integrate the automatic program installer, which would install the latest version of the selected free programs on the desired system.

Storing the saved profiles could be accomplished even in a cloud-based system that is gaining more and more space today. Using a cloud-based service would mean that we would restore an account that we had already saved anywhere in the world. The possibility of further development depends on the user needs. 


\section{CONCLUSION}

Home oriented migration tools surely have a place in our daily lives, it makes the system change easier, however it has some contenders on the market and some limitations as well. The service's greatest contenders are not the migrating software, instead the cloud storage services. If people can store all their data in cloud services, they don't really need to make backups anymore.

Nowadays it's impossible to store every personal data and settings in a cloud service, but the future is heading that way. Even then, cloud services will have their own limitations and disadvantages, this profile managing software is justifiable for a long time.

Further developments have a lot of potential. The application can include an installer part, where open source programs could be selected and installed automatically, which would improve the readiness of the new system even more. Using cloud services would help to migrate the data globally, which is an amazing solution. The possibilities are endless, only resources can limit the final product.

\section{ACKNOWLEDGEMENT}

Authors would like to thank to the University of Pannonia's (Hungary, Veszprem) Neumann Talent Care Program and the Arconic-Köfém Ltd. for their support in testing process.

Authors acknowledge the financial support of Széchenyi 2020 under the EFOP-3.6.1-16-2016-00015.

\section{REFERENCES}

Aomei (2018). AOMEI Softwer backup. https://www.backup-utility.com/

AppiXoft (2018) AppiXoft User Profile Management - https://www.appixoft.com/user-profile-management/

Bila N. et al. (2012). Efficient idle desktop consolidation with partial VM migration. In Proceedings of the 7th ACM European Conference on Computer Systems (EuroSys '12). ACM, New York, NY, USA, 211-224. DOI=http://dx.doi.org/10.1145/2168836.2168858

EU (2018) General Data Protection Rules - http://data.europa.eu/eli/reg/2016/679/oj 2018.

Jayathilake D. (2015) Windows User Profile Management - https://gallery.technet.microsoft.com/Windows-user-profile43679451

Kupczyket M. et al. (2005) Applications on demand" as the exploitation of the Migrating Desktop. Future Generation Computer Systems Volume 21, Issue 1, 1 January 2005, Pages 37-44

PCMover (2018). PCMover Express - https://web.laplink.com/pcmover_profile_migrator/\#ppm-about

Young D. (2013). Software Development Methodologies. White paper.

Rankin J. (2016) Virtualizing Windows10 User Profiles - https://www.htguk.com/everything-you-wanted-to-knowabout_26/

User profiles. (2018.) Microsoft Windows User Profiles -- $\mathrm{https}: / / \mathrm{msdn}$.microsoft.com/enus/library/windows/desktop/bb776892(v=vs.85).aspx

V-model software development and lifecycle (2016) - http://www.professionalqa.com/butterfly-model

Zima D, 2015. Modern Methods of Software Development, TASK Quarterly, Vol. 19, No. 4, 2015. 